\title{
PERCEPCIÓN FAMILIAR Y ACTUACIONES EN LAS SITUACIONES DE FINAL DE LA VIDA DE PERSONAS CON DISCAPACIDAD INTELECTUAL
}

\section{Family Perception and Actions to Take in Situations of End of Life of People with Intellectual Disability}

\author{
Pako Mendizabal Agirre \\ Uliazpi. Guipúzcoa \\ pmendizabal@gipuzkoa.eus \\ Alberto Merino Laca \\ Uliazpi. Guipúzcoa \\ Josune Martínez Alonso \\ Uliazpi. Guipúzcoa \\ Ana Corrales Recio \\ Uliazpi. Guipúzcoa \\ Purificación Cazón del Canto \\ Uliazpi. Guipúzcoa
}

Recepción: 23 de octubre de 2019

Aceptación definitiva: 24 de marzo de 2020

RESUMEN: Las personas con discapacidad intelectual pueden necesitar una planificación centrada en los procesos de final de la vida para una muerte digna en situaciones de enfermedad avanzada, progresiva e incurable. El objetivo del estudio ha sido conocer las percepciones, actitudes, valores y creencias de las familias y sistematizar las actuaciones de los profesionales en los procesos de final de la vida de las personas usuarias del servicio de vivienda de Uliazpi. Se ha diseñado y aplicado una encuesta a tutores/familiares de las personas usuarias sobre diferentes aspectos de este tema. Los resultados del estudio muestran un deseo claro de participar en el proceso, de recibir cuidados paliativos cuando sean necesarios y de acompañar a su familiar en condiciones de intimidad. A partir de estos resultados, se han identificado actuaciones para el apoyo y acompañamiento de las personas usuarias y de sus familias y tutores, en coherencia con la Ley vasca de Garantía 
de los Derechos y de la Dignidad de las personas en el proceso final de la vida y con las preferencias y valores de las personas usuarias y de sus tutores y familiares.

Palabras Clave: final de la vida; percepción familiar; actuaciones; toma de decisiones; cuidados paliativos.

Aвstract: Persons with intellectual disabilities may need plans for dignified endof-life processes in situations of advanced, progressive and incurable illness. The aim of the study has been to learn the perceptions, attitudes, values and beliefs of their families and systematize the actions to be taken by professionals in the end-of-life processes of users of Uliazpi's housing service. A survey has been drawn up on different aspects of this topic and given to users' tutors/families. The results of the survey show the desire to participate in the decision making, to receive palliative care when necessary and to accompany their relatives in conditions of intimacy. With these results, actions have been identified for the support and accompaniment of users and their families/tutors in accordance with the Basque Law on the Guarantee of Rights and Dignity of Persons in the End-of-Life Process, taking into account users' preferences and values and those of their tutors and relatives.

KEY WORDs: end of life; family perception; actions; decision making; palliative care.

\section{Introducción}

$\tau$

as Personas con Discapacidad Intelectual (PcDi) necesitan planes personales para vivir una vida plena y de calidad. Asimismo, cuando es posible anticiparlo, también pueden necesitar una planificación centrada en los procesos de final de la vida o para una muerte digna en situaciones de enfermedad avanzada, progresiva e incurable (otras veces, la muerte llega de forma inesperada y se hacen imposibles la planificación y la despedida previa). Esta planificación centrada en los procesos de final de la vida es plenamente coherente con el enfoque de la "Planificación Personal por Adelantado” (PPA), basada en el trabajo de Creaney Kingsbury (2009).

Dicha "Planificación por Adelantado" tiene como objetivos (citado en Carratalá, Mata y Crespo, 2017, p. 12):

- mantener la dignidad y el respeto por el que se ha luchado toda la vida,

- ayudar a la gente a hacer vida propia y a tomar decisiones para disfrutar de su propia y personal calidad de vida,

- encontrar el equilibrio entre lo que es importante para la persona y lo que hay que hacer por la persona,

- asegurar que se tiene información sobre lo que es "vivir bien" para la persona,

- asegurar que las personas mantienen un control positivo sobre su vida y sobre el final de su vida.

La PPA es un enfoque más de Planificación Centrada en la Persona y comparte, por tanto, los mismos principios y estrategias, pero con la aportación específica de 
incluir la etapa final de la vida como parte de la planificación ya que "al igual que cada uno queremos vivir de una manera, también queremos envejecer o morir a nuestra propia manera" (Carratalá, Mata y Crespo, 2017). Este enfoque cobra una creciente mayor importancia ya que, como dice Cithambaram (2017),

la esperanza de vida de las personas con discapacidad intelectual ha aumentado en los últimos años. Debido a ello, estas personas están experimentando las mismas enfermedades que las personas sin discapacidad tales como, por ejemplo, demencias, cáncer, enfermedades cardíacas, accidentes cerebro-vasculares, etc. De esta manera, también ellas requieren el mismo cuidado y apoyo en las situaciones de final de la vida que las demás personas.

La OMS ha identificado el colectivo de Personas con Discapacidad Intelectual como desventajado, vulnerable y marginalizado (Davis y Higginson, 2004), experimentando desigualdad en el acceso a servicios sanitarios y falta de formación por parte de los profesionales de los servicios sociales sobre los programas de cuidados paliativos y otros similares o relacionados. Según Cithambaram,

puede haber muchas barreras dificultando que las PcDI puedan recibir un adecuado apoyo y cuidado en las situaciones de final de la vida: limitaciones sociales y comunicativas de las propias personas, posible fragmentación de los apoyos y cuidados que reciben las personas, la cultura organizativa de las entidades que prestan apoyos a dichas personas, la falta de información y formación de los profesionales, ausencia de coordinación entre profesionales sociales y sanitarios, etc.

Probablemente se pueda hacer mucho más en lo que se refiere a formación y coordinación de los profesionales, así como en favorecer la implicación y participación de las PcDI en estos procesos. También lo expresan así McCallion et al. (2017) y recomiendan realizar esfuerzos para la formación especializada de los profesionales en cuestiones de final de la vida, para la coordinación de profesionales de apoyo a la DI y de cuidados paliativos y para favorecer la implicación temprana de las propias personas con DI (y/o de sus tutores) en la planificación de las etapas avanzadas de la vida.

Respecto a la formación de profesionales, Tuffry-Wijne, Rose, Grant y Wijne (2017) consideran que el miedo del personal, las influencias culturales y la inexperiencia en relación con las conversaciones en torno a la muerte son barreras comunicativas importantes en la relación con las personas usuarias y, por tanto, que los servicios de DI deben tener estrategias claras de formación a los profesionales acerca de la muerte. Lord, Field y Smith (2017) opinan que es esencial una cultura más abierta en torno a cuestiones sobre la muerte, el duelo, etc., en los contextos de discapacidad intelectual y debe promoverse a través de la formación y el apoyo del personal. Wiese, Stancliffe, Blandin, Howarth y Dew (2012) sondearon la visión del personal respecto a 5 cuestiones críticas: el conocimiento/experiencia sobre la muerte, los valores éticos, el lugar del cuidado, el cómo del cuidado y el cuidado postmuerte, y encontraron que el apoyo en el final de la vida representa un tema complejo pero muy necesario. Grindod, Rumbold y Varney (2017) proponen varias recomendaciones: 
- que se pongan en marcha estrategias para promover la concienciación y clarificar el papel que juega el personal en el apoyo a PcDI en el final de la vida,

- que se identifiquen y se creen los recursos necesarios para proporcionar los apoyos en el final de la vida,

- que se favorezca el proceso de toma de decisiones entre todos los agentes implicados (propia persona, familia, coresidentes cuando sea apropiado, personal, etc.).

Respecto a la necesidad de coordinación, al igual que McCallion et al. (2017), Grindod y Rumbold (2015) insisten en este aspecto de la colaboración de servicios sociales y cuidados paliativos y comentan que "las cuestiones sobre el final de la vida requieren la colaboración de los servicios sociales de discapacidad y los sanitarios de cuidados paliativos y dicha colaboración debe ser liderada por los servicios de discapacidad".

Respecto a la implicación y participación de las personas con DI ( $y$ de sus tutores), Read y Cartlidge (2012) encontraron que a las PcDI no se les daba oportunidad de expresar sus preferencias en relación con diversos aspectos en relación con el final de la vida (lugar, funeral, etc.). En la práctica, las PcDI reciben habitualmente un apoyo y un cuidado pobre, inadecuado e inapropiado en cuestiones relacionadas con el final de la vida (Dunkley y Sales, 2014; McLaughlin, Barr, McIlfatrick y McConkey, 2014; Tuffrey-Wijne, 2009...). Dado que las PcDI tienen los mismos derechos que el resto de la población, deberían tener más oportunidades para discutir y dictar sus propias necesidades en las cuestiones sobre el final de la vida. Existen herramientas, además, que evalúan y facilitan la participación de personas con DI en las situaciones de final de la vida (Stancliffe, Wiese, Read, Jeltes y Clayton, 2017) y, concretamente, para el uso con personas con DI y que evalúan la comprensión del concepto de muerte, el miedo a la muerte y el grado posible de implicación en la planificación en el final de la vida. Algunos de estos instrumentos son los siguientes:

- El Cuestionario sobre el Concepto de la Muerte (CODQ, McEvoy, McHale y Tierney, 2012) evalúa la comprensión del concepto de muerte y sus características de causalidad, irreversibilidad, cesación-no funcionalidad, inevitabilidad y universalidad.

- La Escala de Planificación del Final de la Vida (EOLPS, Wiese, Stancliffe, Clayton, Read y Jeltes, 2014) cuenta con 7 subescalas referidas a otros tantos aspectos relacionados con las decisiones a tomar en el final de la vida: donaciones, lugar preferido de cuidado, deseos en torno al funeral o similar, cuidadores preferidos, anticipación de cuidados, "cosas que llevarme”, donación de órganos.

- La Escala de Miedo a la Muerte (FODS 3.0, Lester y Abdel-Khalek, 2003), específica para PcDI, evalúa el miedo a la muerte propia y a la de otras personas.

En el aspecto específico de los cuidados paliativos, la Asociación Europea de Cuidados Paliativos para PcDI dispone una serie de normas internacionales de consenso en el documento "Consensus norms for palliative care of people" (Tuffrey-Wijne y McLaughlin, 2015). Algunas de ellas son las siguientes: 
- Se deben poner los medios para poder reconocer la necesidad de cuidado paliativo en una PCDI, se debe garantizar la misma oportunidad de acceso a los mismos que el resto de la población y, si es necesario, desarrollar servicios específicos para dicha población.

- Debe promoverse la formación especializada para los profesionales de apoyo.

- Debe promoverse la colaboración entre profesionales y servicios implicados (centros de discapacidad y servicios sanitarios de cuidados paliativos y otros).

- Se deben considerar las necesidades de comunicación de las PcDI y se deben prestar los apoyos necesarios para ello.

- Se deben evaluar todas las necesidades de la persona, tanto físicas, como emocionales, sociales y espirituales, de manera similar al resto de la población.

- Se deben reconocer las posibles mayores dificultades de evaluación de dolor y otros síntomas, y colaborar personas que conocen bien a la persona y los profesionales del control de los síntomas, para una mejor gestión de los mismos.

- Debe evitarse la sobreprotección y ofrecer educación sobre la muerte para las PcDI. Las PcDI deben implicarse al máximo en la planificación y en la toma de decisiones, con los apoyos que puedan necesitar, cuando sea apropiado y deseado.

- Deben implicarse también en el proceso otras personas significativas para la persona (familiares, amistades, personas de apoyo cercanas, etc.).

- Debe contemplarse el apoyo a familiares y personas de apoyo.

- Deben ofrecerse apoyos para el duelo y oportunidades para la participación en funerales y otros actos de recuerdo.

Por otro lado, muy recientemente se ha propuesto una herramienta de cribado (Vrijmoeth et al., 2018) para identificar a las personas que puedan estar en una situación de conveniencia o necesidad de cuidados paliativos. El instrumento evalúa 39 ítems referidos a aspectos relacionados con aspectos físicos, actividades, conductas, síntomas, infecciones, fragilidad, enfermedades y diagnóstico/pronóstico. Aunque no ofrece una puntuación de corte, puede facilitar la explicitación de las dificultades crecientes y el diálogo necesario del equipo multidisciplinar de profesionales con la persona usuaria y/o con su familia para la toma de decisiones en relación a la conveniencia de recabar la colaboración de los equipos de cuidados paliativos y su inclusión en el programa correspondiente.

También hay instrumentos para evaluar la calidad del proceso de final de la vida y la muerte (Downey et al., 2010; Pérez-Cruz et al., 2017; Engelberg et al., 2010) desde el punto de vista de diferentes agentes para evaluar la actuación del profesional en estas situaciones. Si bien no son herramientas específicas para utilizarlas en el ámbito de la discapacidad intelectual, pueden considerarse para evaluar e identificar posibles acciones de mejora en la planificación de estos procesos.

Todo lo anterior sugiere seguir trabajando también en nuestro contexto más cercano para dignificar los procesos de final de la vida de las personas con discapacidad intelectual y del desarrollo.

Uliazpi es una entidad pública cuya actividad es proporcionar servicios y apoyos que contribuyan al desarrollo de la calidad de vida de las personas con discapacidad 
intelectual y sus familias en Guipúzcoa. La inmensa mayoría de las personas usuarias de sus servicios presentan necesidades muy significativas de apoyo. Se observa, en los últimos años, que la población atendida va envejeciendo: actualmente, el $82 \%$ de la totalidad de las personas usuarias de los servicios de vivienda y atención de día tiene más de 40 años, casi el 54 \% tiene más de 50 años y casi el 16 \% tiene más de 60 años, con una edad media aproximada de 48 años. Ante esta coyuntura, los profesionales se van encontrando con más situaciones y procesos de final de la vida y fallecimientos de dichas personas y se espera que vaya ocurriendo con mayor frecuencia en los próximos años. Tal y como se puede ver en el gráfico adjunto, el número de fallecimientos va aumentando ligeramente en los últimos 10 años. La edad media de las personas fallecidas, en cambio, aumenta de forma más notoria. Así, por ejemplo, en 2018 un total de 8 personas usuarias del servicio de vivienda fallecieron (de un total de 289), siendo la edad media de 56 años, lo que contrasta con los datos del estudio de Arvio, Salokivi y Bjelogrlic-Laakso (2016), en el que se habla de una edad media de 44 entre la población con discapacidad intelectual severa y profunda. En la Figura 1, se puede ver la evolución del número de fallecimientos y la de la edad media de los mismos.

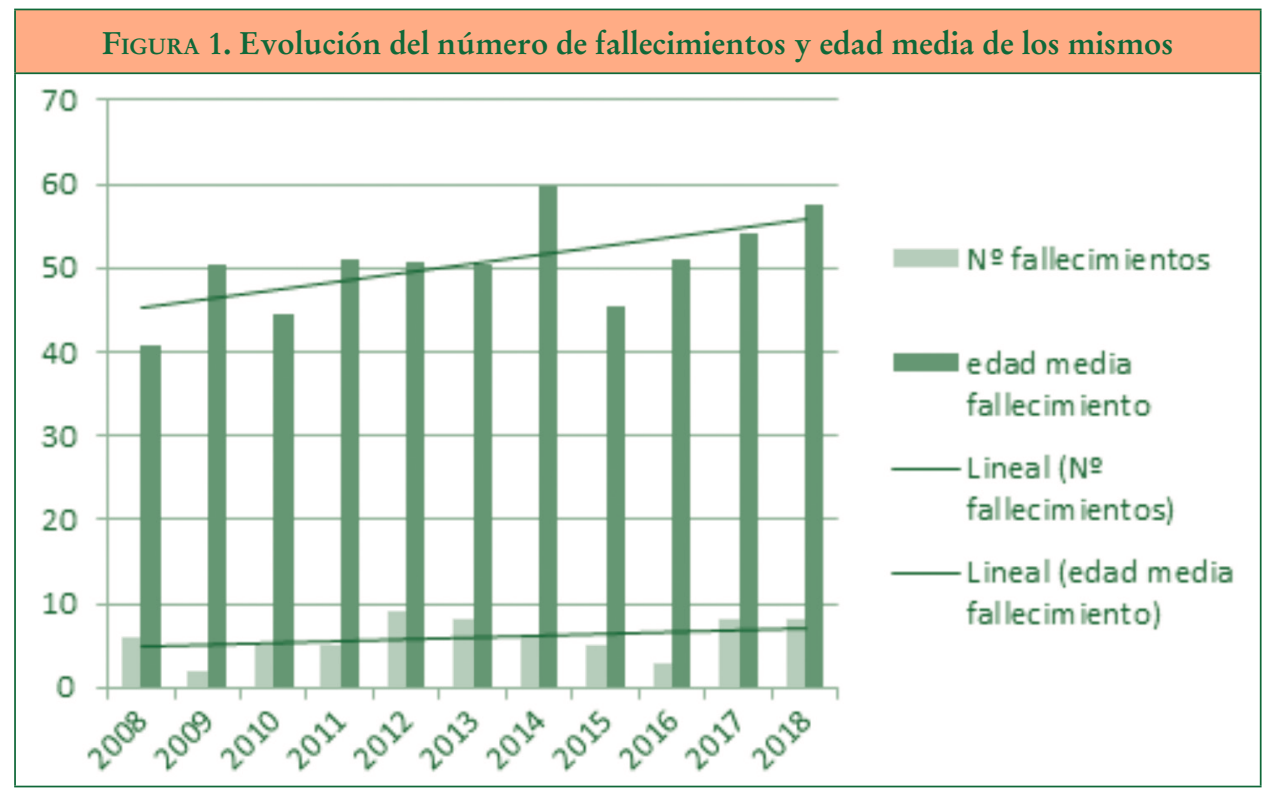

En estas situaciones, los diversos profesionales implicados se han ido conduciendo en cada caso y momento con buena intención y sentido común, pero quizás sin una referencia clara y sistemática de actuación. Ante ello, se impone la reflexión y se hace indispensable sistematizar y explicitar las actuaciones a realizar por parte de los profesionales para apoyar y acompañar a las personas en estos procesos y garantizar sus derechos y dignidad en los procesos de final de la vida. Con este trabajo lo que 
se quiere conseguir es disponer de un protocolo que oriente las actuaciones de los profesionales de Uliazpi para el apoyo y el acompañamiento de las persona usuarias y de sus familias o tutores. Asimismo estará disponible para que profesionales de otras entidades puedan tenerlo como referencia si así lo estiman oportuno.

Para el desarrollo de este trabajo ha sido muy oportuna la promulgación de normativa específica sobre este tema realizada en el País Vasco. Se trata de la Ley 11/2016, de 8 de julio, de Garantía de los Derechos y de la Dignidad de las Personas en el Proceso de Final de su Vida. En ella, se exponen los principios básicos, los derechos de las personas, las obligaciones de los profesionales y las garantías que deben ofrecer los centros sociales y sanitarios de atención (Gobierno Vasco, 2016).

Con la referencia de la citada normativa, se configuró en Uliazpi un equipo de trabajo multidisciplinar entre profesionales de distintos centros y diversas categorías profesionales para elaborar un protocolo de actuación ante estas situaciones de final de la vida de las personas usuarias de sus servicios. Para poder identificar y confirmar las debidas actuaciones a incluir en el protocolo, se decidió, en primera instancia, conocer las percepciones, actitudes, valores y creencias de las personas tutoras (representantes de las personas usuarias) en relación con este tema. Para ello se diseñó y aplicó un cuestionario a familiares y tutores de personas usuarias del servicio de vivienda de Uliazpi de edades avanzadas que pudiesen justificar una relativamente próxima situación de final de la vida. Por medio de la misma, se trataba de determinar cómo ven y desean vivir el final de la vida del familiar, qué apoyos creen que necesitarán, dónde creen que es mejor que esté el familiar, etc., preguntas y respuestas que pueden dar luz para dar apoyos adecuados en estas situaciones a las propias personas con DI, a sus familiares y a los/las profesionales del servicio de vivienda de Uliazpi.

El objetivo del trabajo ha sido conocer la percepción familiar y las actitudes, valores y creencias de las familias sobre las situaciones de final de la vida para, posteriormente, poder identificar las actuaciones más apropiadas por parte de los profesionales para apoyar y acompañar a las personas usuarias y a sus tutores y familiares, garantizando sus derechos y dignidad en los procesos de final de la vida, en coherencia con la normativa mencionada (Ley 11/2016).

\section{Metodología}

El trabajo ha consistido en un estudio tipo encuesta acerca de las actitudes, los valores y las creencias de las familias sobre las situaciones de final de la vida, en base a los instrumentos de recogida de datos de cuestionario y entrevista complementaria.

\subsection{Instrumentos}

Por un lado, se diseñó un cuestionario de 18 ítems (ver en el Anexo 1 el listado completo de ítems) para tutores o familiares de personas con discapacidad intelectual usuarias del servicio de vivienda de Uliazpi mayores de 60 años y mayores de 50 en el caso de personas con síndrome de Down. El formato era de respuesta cerrada ("Sí/ 
No/No sé”) salvo en algún caso que era de carácter abierto (ítems 13b, 14, 17 y 18) o de respuesta categorizada con varias alternativas como "en casa”, "en el centro”, "en el hospital” (ítems 4, 5 y 10).

Por otro lado, de manera complementaria, se realizaron entrevistas individuales, con ítems de respuesta abierta, a una muestra de tutores de personas usuarias fallecidas en los dos últimos años de acuerdo a diversas cuestiones a evaluar. Para ello, se adaptaron y simplificaron los instrumentos de Downey et al. (2010) para evaluar la calidad del proceso de final de la vida en los diferentes casos. Fundamentalmente, se evaluaron las siguientes 5 cuestiones:

- ¿En qué medida te sentiste informado/a en el proceso?

- ¿En qué medida te sentiste acompañado/a en el proceso?

- ¿Qué agradeciste especialmente?

- ¿Qué echaste de menos?

- ¿Qué tipo de apoyo consideras que es importante en esos momentos?

\subsection{Procedimiento}

Se envió el cuestionario a la muestra de tutores junto con una carta explicativa y un sobre franqueado para facilitar la respuesta. Además, se contactó telefónicamente en todos los casos para explicarles personalmente el objetivo y los contenidos de la encuesta.

Para las entrevistas individuales se promovieron encuentros presenciales con cada una de las personas tutoras. Todas las personas concedieron su consentimiento previo informado para la participación en el estudio.

\subsection{Participantes}

Tutores o familiares de personas con discapacidad intelectual usuarias del servicio de vivienda de Uliazpi mayores de 60 años y mayores de 50 en el caso de personas con síndrome de Down. La muestra total era de 46 tutores y se envió el cuestionario a todos ellos. El número de encuestas recogidas fue de 35 , lo que supone un 76,1 \% de las encuestas enviadas.

Por otro lado, fueron 10 las personas tutoras con las que se hicieron las entrevistas complementarias de respuestas abiertas sobre su percepción del proceso a posteriori.

\section{Resultados}

3.1. Resultados del cuestionario a las personas tutoras de las personas usuarias del servicio de vivienda

El 97,1 \% de las personas tutoras ha pensado alguna vez en el fallecimiento de su familiar con discapacidad intelectual, al 82,3\% le parece conveniente reflexionar y planificar el futuro y el $100 \%$ considera necesario participar en la toma de decisiones sobre ello. 
Sobre el lugar más conveniente para los últimos momentos, al 72,7 \% le gustaría que los pasase en el centro donde vive (que ya consideran su casa) y el 65,6 \% además cree que así le gustaría a su familiar.

Sobre la preparación, información y formación sobre la muerte, se aprecian dudas en el $42 \%$ en relación con la preparación de las personas con discapacidad intelectual, el 54,3 \% la considera conveniente en su caso y lo tiene claro respecto a la formación específica de los profesionales (el $80 \%$ la considera necesaria).

En relación con los cuidados paliativos, al 82,9 \% le parecería correcto que su familiar los recibiese, al 77,4 \% le parecería oportuno que los recibiese en el centro donde viven habitualmente, el 91,5 estaría en contra de que se utilizasen tratamientos que solo alarguen la vida de su familiar y el 94,4\% desearía que el tratamiento estuviese limitado a medidas que mantegan a la persona cómoda y aliviada de dolor y sufrimiento.

En relación con los apoyos que podrían necesitar en estas situaciones, el 45,4\% considera que los necesitaría y el 83,3\% considera que los apoyos deberían ser de tipo informativo, el $40 \%$ de tipo emocional y el 5,7 \% de tipo espiritual o religioso.

Finalmente, por lo que respecta a las condiciones más adecuadas para esos últimos momentos, el 88,2 \% considera importante que su familiar pueda estar en una habitación individual y al $100 \%$ le gustaría que estuviese acompañada de algún familiar cercano.

\subsection{Resultados de las entrevistas individuales a tutores de personas fallecidas}

El $90 \%$ de las personas entrevistadas se sintieron adecuadamente informadas del proceso de final de la vida de su familiar. Solo el 10 \% comentó que la información de los aspectos de salud pudo ser más completa.

El $100 \%$ de las personas entrevistadas se sintieron arropadas y apoyadas en todos los momentos del proceso por parte de todos los profesionales del centro, especialmente por las personas de apoyo directo.

El $100 \%$ de las personas entrevistadas agradecieron especialmente el apoyo emocional, el calor y trato humano y la compañía permanente (expresado de diferentes maneras y con diversos matices).

El $60 \%$ de las personas entrevistadas no echaron de menos nada, pero el $20 \%$ de ellas echaron en falta la presencia de otros familiares en el proceso, un $10 \%$ sintió que interfería su presencia en la vida habitual del centro y otro $10 \%$ más echó de menos la posibilidad posterior de despedirse de otras familias.

El $100 \%$ de las personas entrevistadas consideraron importante el apoyo emocional, el cariño y la compañía de los profesionales y un $10 \%$ de ellas añadieron el apoyo en las decisiones y trámites posteriores al fallecimiento del familiar.

\section{Conclusiones}

Se ha conseguido, al menos parcialmente en lo referido a las cuestiones incluidas en el cuestionario, conocer la percepción familiar y las actitudes, los valores y las creencias de 
las personas tutoras de una muestra de personas usuarias del servicio de vivienda de Uliazpi y, sobre la base de ello, identificar algunas posibles actuaciones para el apoyo y acompañamiento en los procesos de final de la vida. Los resultados apuntan a que las personas tutoras piensan en el futuro de sus familiares con discapacidad intelectual y creen conveniente su participación en el proceso de toma de decisiones apoyando o representando a sus familiares. Del análisis de los resultados se pueden extraer algunas conclusiones a considerar, con las que se ha elaborado el protocolo de actuación (en Anexo 2) para guiar el trabajo de los profesionales:

- Es conveniente ir anticipando la reflexión sobre los posibles procesos de final de la vida de sus familiares con DI entre los familiares y personas tutoras, así como ir planificando por adelantado algunas decisiones en relación con ello.

- Es importante planificar estas situaciones y orientar las actuaciones de los profesionales de apoyo mediante procedimientos específicos (final de la vida, defunción, duelo, etc.).

- Es importante considerar el derecho a la información de las PcDI y de sus familias y adaptar esta en la medida de lo posible para afrontar los procesos de final de la vida y poder tomar las decisiones oportunas con el debido apoyo.

- Es importante establecer mecanismos de formación específica para los profesionales, así como apoyos concretos para afrontar estas situaciones.

- Es fundamental establecer relaciones de colaboración con los equipos sanitarios específicos ("Cuidados Paliativos", "Hospitalización a Domicilio", "Paciente Frágil”, etc.) para poder afrontar adecuadamente los procesos de final de la vida de las personas usuarias de nuestros servicios.

- Es necesario implementar actuaciones de apoyo y de acompañamiento a las personas y a las familias en estas situaciones.

- Es imprescindible facilitar el uso de habitaciones individuales y el acompañamiento de las familias en los últimos momentos de las personas.

- Se refuerzan los contenidos y las actuaciones contempladas en el procedimiento de actuación ante procesos de final de la vida elaborado por un equipo de profesionales de Uliazpi y en coherencia con la Ley vasca de "garantía de los derechos y de la dignidad de las personas en el proceso final de su vida”.

En relación con otros trabajos, se han encontrado escasos estudios que evalúen la percepción familiar de los procesos de final de la vida en personas con discapacidad intelectual, máxime en el caso de aquellas con necesidades de apoyo significativas, en el que la participación de los familiares o tutores es más relevante, por representación o sustitución en la toma de decisiones (Etxeberria, 2016). Sí existen, por el contrario, estudios sobre la percepción de los profesionales (Grindo, Rumbold y Varney, 2016; Lord, Field y Simyh, 2017; Wiese et al., 2012) y alguno sobre la de las propias personas (Watson, Wilson y Hagiliassis, 2017). Sobre percepción familiar, cabe citar el estudio de Cithambaram (2017), en el que llama la atención sobre la importancia de la adecuada información a las personas con discapacidad intelectual y a sus familiares, así como de facilitar un alto nivel de "familiaridad" en las condiciones de dichos procesos. 
Se constatan algunas limitaciones del presente estudio, el cual se centra en la percepción de familiares y tutores de personas usuarias del servicio. Aunque, de cara a la elaboración del protocolo, se ha contado con un equipo de profesionales, el punto de vista de los profesionales no queda recogido de manera amplia y profunda. Igualmente, motivado por las muy significativas necesidades de apoyo de la población atendida en Uliazpi, no ha sido posible considerar la perspectiva de las propias personas con discapacidad intelectual, indudablemente muy relevante en este tema. Por otro lado, la muestra de personas tutoras ha sido de 35 en el caso de la aplicación de los cuestionarios escritos y de 12 en el caso de las entrevistas. Sin duda, un mayor tamaño de las muestras hubiese arrojado resultados más significativos. Finalmente, el estudio se ha centrado preferentemente en los momentos previos a las situaciones de fallecimiento de las personas, incidiendo solo tangencialmente en aspectos relacionados con las situaciones posteriores.

De las limitaciones del estudio apuntadas, se puede derivar la identificación de posibles líneas de investigación. Por un lado, sería deseable revalorar la percepción familiar con una muestra mayor de personas y con un abanico más amplio de cuestiones. Asimismo sería muy oportuno evaluar las perspectivas de las propias personas con discapacidad intelectual y/o del desarrollo, así como la de los profesionales (sociales y sanitarios) implicados.

\section{Referencias bibliográficas}

Arvio, M., Salokivi, T. y Bjelogrlic-Laakso, N. (2016). Age at death in individuals with intellectual disabilities. Journal of Applied Research in Intellectual Disabilities, 30, 1-4. Disponible en https://doi.org/10.1111/jar.12269

Carratalá, A., Mata, G. y Crespo, S. (2017). Planificación centrada en la persona: planificando por adelantado el futuro deseado. Madrid: Plena inclusión. Disponible en http://www. plenainclusion.org/sites/default/files/guia_planificacion_plena_inclusion_completob.pdf.

Cithambaram, K. (2017). Preserving self in the face of death and dying: a grounded theory of end of life care needs of people with intellectual disabilities. Tesis doctoral Universidad de Dublín. Disponible en http://doras.dcu.ie/21630/1/Kumaresan_Cithambaram_2017_ PhD_thesis.pdf.

Creaney Kingsbury, L. A. (2009). People Planning Abead. A guide to communicating healthcare and end of life wishes. Washington: American Association in Intellectual and Developmental Disabilities (AAIDD).

Davies, E. e Higginson, I. (2004). Better palliative care for older people [Online]. Disponible en http://www.euro.who.int/_data/assets/pdf_file/0009/98235/E82933.pdf

Domingo, E., Cuesta, J. L. y SÁnchez, S. (2018). Cómo afrontar el duelo en las personas con discapacidad intelectual. Una aproximación al problema. Siglo Cero, 49(4), 51-68. Disponible en http://dx.doi.org/10.14201/scero20184945168

Downey, L., Curtis, J. R., Lafferty, W. E., Herting, J. R. y Engelberg, R. A. (2010). The quality of dying and death questionnaire (QODD): empirical domains and theoretical persepectives. Journal of Pain and Sympton Management, 39, 9-22. Disponible en https:// doi.org/10.1016/j.jpainsymman.2009.05.012 
Dunkley, S. y Sales, R. (2014). The challenges of providing palliative care for people with intellectual disabilities: a literature review. International Journal of Palliative Nursing, 20(6), 279-284. Disponible en https://doi.org/10.12968/ijpn.2014.20.6.279

Engelberg, R. A., Downey, L., Wenrich, M. D., Carline, J. D., Silvestri, G. A., DotoLo, D., Nielsen, E. L. y Curtis, J. R. (2010). Measuring the quality of end-of-life care. Journal of Pain and Symptom Management, 39(6), 951-971. Disponible en https://doi. org/10.1016/j.jpainsymman.2009.11.313

ETXEBERria, X. (2016). Autonomía y decisiones de representación/sustitución en personas con discapacidad intelectual. Perspectiva ética. Siglo Cero, 47(1), 55-66. Disponible en http:// dx.doi.org/10.14201/scero201615566

Gobierno Vasco. (2016). Ley 11/2016 de 8 de julio, de Garantía de los Derechos y de la Dignidad de las Personas en el Proceso Final de la Vida. Boletín Oficial del País Vasco, 14 de julio de 2016, núm. 134, 1-16. Disponible en http://www.euskadi.eus/bopv2/ datos/2016/07/1603138a.pdf.

Grindod, A. y Rumbold, B. (2017). Providing end of life care in disability community living services: an organizational capacity-building model using a public health approach. Journal of Applied Research in Intellectual Disabilities, Special Issue (00), 1-13. Disponible en https://doi.org/10.1111/jar.12372

Grindod, A., Rumbold, B. y VARney, H. (2015). End of life and palliative care project: people with intellectual disabilities living in residenctial services. Research report, Melbourne, Australia: La Trobe University Palliative Unit. Disponible en https://doi.org/10.13140/ RG.2.2.32456.11527

Janicki, M. P., Watchman, K. y Fortea, J. (2018). Principales conclusiones acordadas durante la cumbre sobre discapacidad intelectual y demencia. Siglo Cero, 49(2), 115-122. Disponible en http://dx.doi.org/10.14201/scero2018492115122

Lester, D. y Abdel-Khalek, A. (2003). The Collect-Lester fear of death scale: a correction. Death Studies, 27(1), 81-85. Disponible en https://doi.org/10.1080/07481180302873

Lord A., Field, S. y SMith, I. C. (2017). The experiences of staff who support people with intellectual disability on issues about death, dying and bereavement: a methasynthesis. Journal of Applied Research in Intellectual Disabilities, 30, 1007-1021. Disponible en https:// doi.org/10.1111/jar.12376

McCallion, P., Hogan, M., Santos, F. H., McCarron, M., Service, K., Stepm, S., Keller, S., Fortea, J., Bishop, K., Watchman, K. y Janicki, M. P. (2017). Consensus statement of the international summit on intelectual disability and dementia related to end of life care in advanced dementia. Journal of Applied Research in Intellectual Disabilities, Special Issue, 1-5. Disponible en https://doi.org/10.1111/jar.12349

McEvoy, J., MacHale, R. y Tierney, E. (2012). Concept of death and perceptions of bereavement in adults with intellectual disabilities. Journal of Intellectual Disability Research, 56(2), 191-203. Disponible en https://doi.org/10.1111/jir.12347

Mclaughlin, D., Barr, O., McIlfatrick, S. y McConkey, R. (2014). Developing a best practice model for partnership practice between specialist palliative care and intellectual disability services: a mixed methods study. Palliative Medicine, 28(10), 1213-1221. Disponible en https://doi.org/10.1177/0269216314550373

Mendizábal, P., Merino, A., Martínez, J., Corrales, A. y Cazón, P. (2018). Planificarpara la vida, planificar para la muerte. Percepción familiar y guía de actuación para profesionales ante los procesos de final de la vida de personas con discapacidad intelectual en Uliazpi. Trabajo Fin del Máster "Apoyos a personas con discapacidad intelectual o del desarrollo y a sus familias: procesos cognitivos y de calidad de vida individual y familiar”. 
Mendizábal, P., Merino, A., Martínez, J., Corrales, A. y Cazón, P. (2018). Planificar para la vida, planificar para la muerte. Comunicación presentada en las X Jornadas Científicas de Investigación sobre Personas con Discapacidad "Estrategias de innnovación y cambio en servicios sociales, educativos y de salud”. Salamanca: INICO. Disponible en Comunicación Salamanca.pptx.

Navas, P., Gómez, L. E., Verdugo, M. Á. y Schalock, R. L. (2012). Derechos de las personas con discapacidad: implicaciones de la Convención de Naciones Unidas. Siglo Cero, 43(3), 7-28.

Navas, P., Verdugo, M. Á., Martínez, S., Sainz, F. y Aza, A. (2017). Derechos y calidad de vida en personas con discapacidad intelectual y mayores necesidades de apoyo. Siglo Cero, 48(4), 7-66. Disponible en http://dx.doi.org/10.14201/scero2017484766

Pérez-Cruz, P. E., Padilla, O., Bonati, P., Thomsen, O., Tupper, L., González, M., CeBallos, D. y Maldonado, A. (2017). Validation of the Spanish version of the quality of dying and death questionnaire (QODD-ESP) in a home based cancer palliative care program and development of the QODD-ESP-12. Journal of Pain and Sympton Management, 53(6), 1042-1049. Disponible en https://doi.org/10.1016/j.jpainsymman.2017.02.005

Read, S. y CARTLIDge, D. (2012). Critical reflections on end-of-life care for people with intellectual disabilities: a single case study approach. International Journal of Palliative Nursing, 18(1), 23-27. Disponible en https://doi.org/10.12968/ijpn.2012.18.1.23

Stancliffe, R. J., Wiese, M. Y., Read, S., Jeltes, G. y Clayton, J. M. (2017). Assessing knowledge and attitudes about end of life: evaluation of three instruments designed for adults with intellectual disability. Journal of Applied Research in Intellectual Disabilities, Special Issue, 1-13. Disponible en https://doi.org/10.1111/jar.12358

Tuffrey-Wijne, I. (2009). The preferred place of care for people who are dying. Learning Disability Practice, 12(6), 16-21. Disponible en https://doi.org/10.7748/ldp2009.07.12.6.16. c7125

Tuffrey-Wijne, I. y McLaughlin, D. (2015). Consensus Norms for Palliative Care of People with Intellectual Disabilities in Europe. EAPC White Paper [Online]. Disponible en http:// www.eapcnet.eu/LinkClick.aspx?fileticket=Iym7SMB78cw\%3D.

Tuffrey-Wijne, I., Rose, T., Grant, R. y Wijne, A. (2017). Communicating about death and dying: developing training for staff working in services for people with intelectual disabilities. Journal of Applied Research in Intellectual Disabilities, 30, 1099-1110. Disponible en https://doi.org/10.1111/jar.12382

Vrijmoeth, C., Echteld, M. A., Assendelft, P., Christians, M., Festen, D., van SchoroJenstein, H., Vissers, K. y Groot, M. (2018). Development and applicability of a tool for identification of people with intellectual disabilities in need of palliative care (Palli). Journal of Applied Research in Intellectual Disabilities, 0, 1-11. Disponible en https://doi. org/10.1111/jar.12472

Wiese, M., Stancliffe, R. J., Balandin, S., Howarth, G. y Dew, A. (2012). End of life care and dying: issues raised by staff supporting older peole with intelectual disability in community living services. Journal of Applied Research in Intellectual Disabilities, 25(6), 571583. Disponible en https://doi.org/10.1111/jar.12000

Wiese, M. Y., Stancliffe, R. J., Clayton, J., Read, S. y Jeltes, G. (2014). End of life planning scale. Centre for Disability Research and Policy, Universitiy of Sydney.

Wilson, J., Wilson, E. y Hagiliassis, N. (2017). Supporting end of life decision making: case studies of relational closeness in supported decision making for people with sever or profound intellectual disability. Journal of Applied Research in Intellectual Disabilities, 00, 1-13. Disponible en https://doi.org/10.1111/jar.12393 


\section{ANEXO 1: \\ Cuestionario para familiares/tutores sobre los procesos de final de la vida}

(Mendizábal, P., Merino, A., Martínez, J., Corrales, A. y Cazón, P., 2018)

\section{PREGUNTAS (Señala con una X la opción elegida)}

1. ¿Has pensado alguna vez en el fallecimiento de tu familiar con discapacidad intelectual?

\begin{tabular}{|l|l|}
\hline Sí & \\
\hline $\mathrm{NO}$ & \\
\hline
\end{tabular}

2. ¿Crees que es conveniente reflexionar sobre ello y planificar dentro de lo posible el proceso?

\begin{tabular}{|l|l|}
\hline SÍ & \\
\hline NO & \\
\hline NO SÉ & \\
\hline
\end{tabular}

3. ¿Crees que es necesario que, como familiares, participéis en las decisiones a tomar en el proceso de final de la vida de tu familiar?

\begin{tabular}{|l|l|}
\hline SÍ & \\
\hline NO & \\
\hline NO SÉ & \\
\hline
\end{tabular}

4. ¿Dónde te gustaría que pasase tu familiar sus últimos días?

\begin{tabular}{|l|l|}
\hline En la casa familiar & \\
\hline En el centro & \\
\hline En el hospital & \\
\hline
\end{tabular}

5. ¿Dónde crees que le gustaría a tu familiar pasar sus últimos días?

\begin{tabular}{|l|l|}
\hline En la casa familiar & \\
\hline En el centro & \\
\hline En el hospital & \\
\hline
\end{tabular}

6. ¿Crees que tu familiar debe saber si se está muriendo?

\begin{tabular}{|l|l|}
\hline SÍ & \\
\hline NO & \\
\hline NO SÉ & \\
\hline
\end{tabular}

Ediciones Universidad de Salamanca / CC BY-NC-ND

Siglo Cero, vol. 51 (3), 2020, julio-septiembre, pp. 27-46 
7. ¿Crees conveniente una preparación sobre la muerte para las personas con discapacidad intelectual?

\begin{tabular}{|l|l|}
\hline SÍ & \\
\hline NO & \\
\hline NO SÉ & \\
\hline
\end{tabular}

8. ¿Crees conveniente una preparación previa sobre la muerte a los familiares de las personas usuarias?

\begin{tabular}{|l|l|}
\hline SÍ & \\
\hline NO & \\
\hline NO SÉ & \\
\hline
\end{tabular}

9. ¿Crees necesaria una formación específica sobre los procesos de final de la vida de las personas usuarias para los profesionales de apoyo?

\begin{tabular}{|l|l|}
\hline SÍ & \\
\hline NO & \\
\hline NO SÉ & \\
\hline
\end{tabular}

10. En caso de estar tu familiar en un proceso de final de su vida ¿te parecería correcto que recibiera cuidados paliativos?

\begin{tabular}{|l|l|}
\hline Sí & \\
\hline NO & \\
\hline NO SÉ & \\
\hline
\end{tabular}

11. En caso de recibir cuidados paliativos ¿¿dónde crees que sería más oportuno recibirlos?

\begin{tabular}{|l|l|}
\hline En la casa familiar & \\
\hline En el centro & \\
\hline En el hospital & \\
\hline
\end{tabular}

12. En el caso de una enfermedad terminal ¿desearías que se utilizasen tratamientos que solamente alarguen la vida?

\begin{tabular}{|l|l|}
\hline Sí & \\
\hline NO & \\
\hline NO SÉ & \\
\hline
\end{tabular}

Ediciones Universidad de Salamanca / CC BY-NC-ND

Siglo Cero, vol. 51 (3), 2020, julio-septiembre, pp. 27-46 
13. ¿Desearías que el tratamiento de tu familiar fuese limitado a medidas que le mantengan cómodo y aliviado de dolor y sufrimiento?

\begin{tabular}{|l|l|}
\hline Sí & \\
\hline NO & \\
\hline NO SÉ & \\
\hline
\end{tabular}

14. ¿Crees que necesitarías algún tipo de apoyo para la toma de decisiones en relación con el final de la vida de tu familiar?

\begin{tabular}{|l|l|}
\hline SÍ ¿Cuál? & \\
\hline NO & \\
\hline NO SÉ & \\
\hline
\end{tabular}

15. ¿Qué apoyos crees que necesitaríais en el proceso de final de la vida de tu familiar? Señala con una $X$ las opciones elegidas (una o más de una)

\begin{tabular}{|l|l|}
\hline Información & \\
\hline Apoyo emocional & \\
\hline Apoyo espiritual/religioso & \\
\hline Otros (¿Cuáles?) & \\
\hline
\end{tabular}

16. ¿Te parece importante que tu familiar pueda estar en una habitación individual en sus últimos momentos?

\begin{tabular}{|l|l|}
\hline SÍ & \\
\hline NO & \\
\hline NO SÉ & \\
\hline
\end{tabular}

17. ¿Te parece importante que tu familiar esté acompañado por algún familiar en sus últimos momentos?

\begin{tabular}{|l|l|}
\hline Sí & \\
\hline NO & \\
\hline NO SÉ & \\
\hline
\end{tabular}

18. ¿Qué otros aspectos consideras importantes en los últimos momentos de la vida de una persona? 
PERCEPCIÓN FAMILIAR Y ACTUACIONES EN LAS SITUACIONES DE FINAL DE LA VIDA DE PERSONAS CON DISCAPACIDAD INTELECTUAL

PAKO MENDIZABAL, ALBERTO MERINO, JOSUNE MARTÍNEZ, ANA CORRALES Y PURIFICACIÓN CAZÓN

19. Otros aspectos relacionados a tener en cuenta que te gustaría añadir.

Ediciones Universidad de Salamanca / CC BY-NC-ND

Siglo Cero, vol. 51 (3), 2020, julio-septiembre, pp. 27-46 


\section{Anexo 2: \\ Procedimiento de actuacion en los procesos de final de la vida}

OBJETO: Orientar las actuaciones de los profesionales de Uliazpi para el apoyo y acompañamiento de las personas usuarias y de sus familias o tutores, garantizando sus derechos y dignidad en los procesos de final de la vida.

Este protocolo toma como referente la Ley vasca 11/2016, de 8 de julio, de garantía de los derechos y de la dignidad de las personas en el proceso final de su vida.

ALCANCE: Este protocolo se refiere a las “personas que se encuentran en el final de la vida”. Según la citada ley, se trata de "personas que presentan un proceso de enfermedad avanzado, progresivo e incurable por los medios tecnológicos existentes, sin posibilidades razonables de respuesta al tratamiento específico y con presencia de sintomatología intensa, multifactorial y cambiante, que condiciona una inestabilidad en la evolución del paciente, así como un pronóstico de vida limitado a corto plazo”.

De forma específica, este protocolo es aplicable a todas las personas que en el proceso final de su vida estén atendidas en el servicio de vivienda de Uliazpi.

\section{RESPONSABILIDADES:}

- Del equipo de salud del centro:

- Informar, junto al RC y RU, al equipo técnico y al PAD del problema de salud detectado, de su evolución y, en su caso, de la irreversibilidad del proceso.

- Informar a la familia o tutor de la evolución e implicaciones de la enfermedad.

- Realizar un seguimiento para detectar cuanto antes los problemas de salud y aplicar los tratamientos de salud adecuados para cada situación y problemática y según el pronóstico estimado.

- Mantener actualizado el historial/expediente de la persona para su posible derivación.

- Derivar o consultar a los equipos especializados de Osakidetza, en función de la situación clínica.

- Del responsable de centro $(\mathrm{RC})$ y unidad $(\mathrm{RU})$ y gobernanta:

- Informar a la familia, acompañarla en todo momento, valorar sus preferencias respecto al modo de afrontar el final de la vida, facilitar su toma de decisiones y recabar su consentimiento en todas las actuaciones.

- Tomar las medidas oportunas en los últimos momentos de vida de la persona (facilitar la intimidad de la persona usuaria y el acompañamiento familiar).

- Informar, dar instrucciones y apoyar a los PAD en los últimos momentos y tras el fallecimiento (procedimiento de defunción).

- Apoyar emocionalmente y acompañar a los familiares en los ultimos momentos. 
- Del personal de atención directa (PAD):

- Observar la evolución de los síntomas y el grado de malestar de la persona enferma e informar de ello al equipo de salud.

DESARROLLO (actuaciones concretas a llevar a cabo en los centros):

PREVIA: En la medida de lo posible, valorar y anticipar en los planes personales de vida de personas en edad avanzada o en situación de especial vulnerabilidad o fragilidad las preferencias personales sobre los diversos aspectos a considerar en los procesos del final de la vida.

A) En el momento del diagnóstico o detección de un problema de salud que puede ser grave:

- Informar al equipo técnico y al PAD (mediante sesión clínica o similar) del problema de salud que presenta la persona.

- Informar a los familiares o tutor del problema de salud y de su posible evolución y pronóstico.

- Planificar los próximos pasos para vigilar la evolución del caso y poder anticipar cambios susceptibles de ser atendidos.

B) En el momento del agravamiento o identificación del problema de salud como incurable e irreversible:

- Solicitar, tras el consentimiento del tutor, la colaboración/derivación a un equipo especializado de Osakidetza ("PAMI”, "Paciente Frágil”, "Hospitalización a domicilio", "Cuidados Paliativos”...) para la atención del caso.

- Informar al equipo técnico y al PAD de la nueva situación.

- Analizar con la familia la nueva situación, valorar su visión sobre ella y anticipar sus preferencias (proporcionando los tiempos necesarios) en relación con las posibles decisiones a tomar (por ejemplo, ingreso hospitatalario o no, soporte vital o no, pruebas invasivas o no, cuidados paliativos o no, etc.) y respetando las mismas.

- Informar, en la medida de lo posible y de forma adaptada, a los compañeros y compañeras más cercanos de la nueva situación.

C) En los últimos momentos previos al fallecimiento de la persona:

- Utilizar, en caso necesario, a propuesta del equipo de salud correspondiente y con el consentimiento informado de la persona tutora, los programas de tratamiento paliativo y de sedación disponibles para el resto de la población.

- Posibilitar un entorno tranquilo e íntimo (habitación individual).

- Facilitar una espacio adecuado para el acompañamiento familiar a la persona usuaria, atendiendo a sus deseos, si es necesario las $24 \mathrm{~h}$ del día y de la noche.

- Proporcionar apoyo emocional a la familia (con los propios profesionales o los del equipo de cuidados paliativos).

- Proporcionar apoyo emocional a los compañeros y compañeras más cercanos. 
- Promover el acompañamiento a la familia por parte de algún profesional (dependiendo del momento del día o noche, RU, RC, enfermero-a, gobernanta-e, PAD...).

- Informar previamente de la posible inminencia del desenlace y apoyar al PAD presente en el momento del fallecimiento (guía de actuación, apoyo emocional, posible liberación, etc.).

D) Tras el fallecimiento de la persona:

- Se considerarán y activarán los procedimientos ya existentes en la organización de actuación en caso de defunción, por un lado, y de actuación en situaciones de pérdida significativa (duelo), por otro.

- En otras circunstancias (fallecimiento en hospital o domicilio) de procesos de final de la vida, se pondrán en marcha las medidas de apoyo o asesoramiento que se consideren oportunas según el caso.

- Realizar alguna actuación de despedida y recuerdo a la persona fallecida y a su familia (carta, vídeo, álbum de fotos...).

- Posibilitar la despedida de la familia con respecto a otras familias del centro.

- Asesorar, en el caso de que lo necesiten, de los trámites posteriores al fallecimiento. 